\title{
Superconducting nanowires: Interplay of discrete transverse modes with supercurrent
}

\author{
M. D. Croitoru, ${ }^{1,2}$ A. A. Shanenko, ${ }^{1}$ C. C. Kaun, ${ }^{2}$ and F. M. Peeters ${ }^{1}$ \\ ${ }^{1}$ Departement Fysica, Universiteit Antwerpen, B-2020 Antwerpen, Belgium \\ ${ }^{2}$ Research Center for Applied Sciences, Academia Sinica, Taipei 11529, Taiwan
}

(Received 30 April 2009; published 22 July 2009)

\begin{abstract}
From a numerical solution of the Bogoliubov-de Gennes equations, we investigate an interplay of the transverse discrete modes with a longitudinal supercurrent in a metallic cylindrical superconducting nanowire. The superconductor-to-normal transition induced by a longitudinal superflow of electrons is found to occur as a cascade of jumps in the order parameter (supercurrent and superfluid density) as a function of the superfluid velocity for diameters $d<10-15 \mathrm{~nm}$ (for Al parameters) and sufficiently low temperatures $T<0.3-0.4 T_{c}$, with $T_{c}$ the critical temperature. When approaching $T_{c}$, the jumps are smoothed into steplike but continuous drops. A similar picture occurs for $d>15-20 \mathrm{~nm}$. Only when the diameter exceeds 50-70 nm the quantumsize cascades are fully washed out, and we arrive at the mesoscopic regime. Below this regime the critical current density $j_{c}$ exhibits the quantum-size oscillations with pronounced resonant enhancements: the smaller the diameter, the more significant is the enhancement. Thickness fluctuations of real samples will smooth out such oscillations into an overall growth of $j_{c}$ with decreasing nanowire diameter.
\end{abstract}

DOI: $10.1103 /$ PhysRevB.80.024513

PACS number(s): 74.78.Na

\section{INTRODUCTION}

Interplay between superconductivity and quantum confinement has been a subject of increasing interest in the last several years. This is motivated by recent experiments on nanosuperconductors. ${ }^{1-7}$ These experiments became possible due to modern technological advances which resulted in the fabrication of high-quality low resistive superconducting metallic nanofilms and nanowires. In most fabricated samples the electron mean-free path $\ell$ was found to scale with the confined dimension $d .^{2,4,6}$ In particular, Matthiessens-like approximation $1 / \ell=1 / 2 d+1 / \ell_{\text {imp }}$ was of use in the second paper of Ref. 2 , where $1 / 2 d$ represents the contribution of the boundaries (i.e., of confinement) and $\ell_{\text {imp }} \gg d$ stands for the mean-free path due to nonmagnetic imperfections. Based on results of this paper, one can estimate that $\ell_{\text {imp }}$ $\sim 200-400 \mathrm{~nm}$ in $\mathrm{Pb}$ single-crystal atomically uniform nanoislands with thickness about $30-40 \AA$ and area $\sim 400 \mathrm{~nm} \times 400 \mathrm{~nm}$. In nanowires disorder is more significant but due to the scaling $\ell \propto d$, we can also expect that the boundary scattering controls $\ell$ and, hence, $\ell_{\text {imp }} \gg d$. Thus from present-day technologies it is possible to fabricate superconducting nanofilms and nanowires with minor disorder, i.e., $\ell_{\text {imp }} / d \gg 1$ and $k_{F} \ell_{\text {imp }} \gg 1$, with $k_{F}$ the three-dimensional (3D) Fermi wave vector. An important consequence is that the discrete transverse electron spectrum is not smeared by impurity scattering in this case, and the conduction band splits up into a series of single-electron subbands which move in energy with changing $d$. As was established half a century ago, 8,9 the superconducting equilibrium properties are not very sensitive to minor disorder. Thus, to first approximation, these high-quality nanosuperconductors can be treated as being in the clean limit. This situation is rather different from the main stream of most previous and current studies of superconductivity in high-resistivity samples with reduced dimensionality that are strongly disordered or granular. In such structures the strong disorder destroys the superconductivity since it enhances the repulsive part of the electron-electron effective interaction and results also in the localization of the electron wave functions (the fermionic mechanism ${ }^{10}$ ) or favor the phase fluctuations of the superconducting condensate (the bosonic mechanism ${ }^{11}$ ). Notice that the high-quality superconducting nanofilms show no significant indications of defect- or phase-driven suppression of superconductivity even down to thicknesses of about a few monolayers. $^{3}$ In high-quality superconducting nanowires clear signatures of the superconducting state are observed even for thicknesses down to $5-8 \mathrm{~nm} .^{4,6}$

The potential application of high-quality nanostructures is another reason for the stimulating active research of their physical and, particularly superconducting, properties. These properties are mainly governed by the size-quantization of the transverse electron spectrum, ${ }^{12}$ which has a substantial impact on the basic superconducting characteristics, e.g., the order parameter, ${ }^{13-16}$ the critical temperature, ${ }^{17,18}$ and the critical magnetic field. ${ }^{19,20}$

One of the most striking (quantum) features of the superconducting state is that the sample offers absolutely no resistance to the flow of an electrical current. The destruction of the supercurrent can occur only after all of its momenta have been transferred to quasiparticle excitations when the individual Cooper pairs are depaired into ordinary itinerant electrons. This process of supercurrent suppression can be viewed as a competition between the Doppler quasiparticleenergy shift $E_{\text {Dopp }}\left(k_{F}\right)=\hbar^{2} k_{F} q / m_{e}$, where $q$ is the superfluid wave vector, and the superconducting energy gap $\Delta_{E}$. Namely, the process of destructing the Cooper pairs starts when the Doppler shift exceeds the superconducting gap. This is the celebrated Landau criterion. ${ }^{21}$ In the $3 \mathrm{D}$ case the superconducting state is not immediately destroyed after the Landau criterion is met; there appears a small region of superfluid velocities with gapless superconductivity (it is possible only for fermions). However only the situation of the supercurrent with the positive derivative with respect to the superfluid velocity can be easily accessible in experiments. ${ }^{22}$ The dimensionality of the sample is of essential importance in the behavior of the superconducting state under current 
flow. As shown in Ref. 23 for the case of one-dimensional (1D) structures, any superconducting quantity immediately drops to zero when $E_{\text {Dopp }}\left(k_{F}\right)=\Delta_{E}$. So, one can expect that quantum confinement has a serious impact on the currentcarrying state in superconducting metallic nanowires. In the presence of the splitting of the conduction band into a series of subbands, a supercurrent flows through a set of 1D quantum channels, and the energy of excitations in any channel depends on the nanowire thickness.

The present paper focuses on a theoretical and numerical study of the superconductor-to-normal transition induced by a longitudinal supercurrent in metallic high-quality nanowires. Our study is based on a numerical solution of the Bogoliubov-de Gennes (BdG) equations for a clean cylindrical nanowire in the presence of quantum confinement for the transverse electron motion. The suppression of the superconducting order by thermal- and quantum-phase fluctuations can be a serious issue in quasi-1D superconducting structures. However, the crossover from the superconducting to normal state due to phase fluctuations is expected in highquality nanowires for thicknesses $\leqslant 5-8 \mathrm{~nm}$ (see the second paper in Ref. 4 and discussion in Sec. V). As to the effects of the transverse quantization, they are of importance when the energy spacing between the transverse levels becomes larger than the bulk superconducting order parameter (at zero temperature), i.e., $\frac{\hbar^{2}}{2 m_{e}} \frac{\pi^{2}}{d^{2}}>\Delta_{\text {bulk }}$. For instance, for Al this is true when $d \lesssim 40 \mathrm{~nm}$.

The present paper is organized as follows. In Sec. II we outline the $\mathrm{BdG}$ formalism for a cylindrical nanowire and express the important current characteristics in terms of the distribution function of quasiparticles. Next, by numerically solving the BdG equations, we study the superconductor-tonormal transition in the presence of a longitudinal supercurrent (Sec. III). The critical current and superfluid density are discussed in Sec. IV. Section V gives our concluding remarks. The details of our numerical algorithm to solve the BdG equations are given in Appendix A. Anderson's approximate semianalytical solution to the $\mathrm{BdG}$ equations is discussed in Appendix B. The basic formula for the superfluid density is derived in Appendix C.

\section{FORMALISM}

To study the critical current and depairing superfluid velocity in high-quality metallic nanowires, we consider a superconducting nanowire with diameter $D$ and length $L$ in the clean limit, as our model system. Due to the transverse quantum confinement, the translational invariance is broken along the confining directions, and the superconducting order parameter is position dependent, i.e., $\Delta(\mathbf{r})$. Here we present a mean-field treatment of such a situation, which is based on the $\mathrm{BdG}$ equations. ${ }^{24}$ In a frame co-moving with the pair condensate (with the superfluid velocity $\mathbf{v}_{s}$ ), the BdG equations for the stationary pseudospinor in the particle-hole space $\left\langle\mathbf{r} \mid \Psi_{i}\right\rangle=\left(\left\langle\mathbf{r} \mid u_{i}\right\rangle,\left\langle\mathbf{r} \mid v_{i}\right\rangle\right)^{\mathrm{T}}$, can be written as

$$
E_{i}\left|u_{i}\right\rangle=\hat{H}_{e}\left|u_{i}\right\rangle+\hat{\Delta}\left|v_{i}\right\rangle,
$$

$$
E_{i}\left|v_{i}\right\rangle=\hat{\Delta}^{*}\left|u_{i}\right\rangle-\hat{H}_{e}^{*}\left|v_{i}\right\rangle,
$$

where $E_{i}$ stands for the quasiparticle (bogolon) energy and the single-electron Hamiltonian (absorbing the chemical potential $\mu$ ) is given by

$$
\hat{H}_{e}=\frac{\hat{\mathbf{P}}^{2}}{2 m_{e}}+V_{\text {conf }}(\hat{\mathbf{r}})-\mu,
$$

where $\hat{\mathbf{P}}=\hat{\mathbf{p}}+m_{e} \mathbf{v}_{s}$ (the band mass $m_{e}$ is set to the freeelectron mass) with $\hat{\mathbf{p}}$ the momentum operator, $\hat{\mathbf{r}}$ is the position operator, and $V(\mathbf{r})$ stands for the confining potential. We remark here that the effects of the magnetic field produced by the supercurrent can be entirely ignored in narrow wires. For the sake of simplicity, the confining interaction $V_{\text {conf }}(\mathbf{r})$ is taken as zero inside the specimen and infinite outside: $V_{\text {conf }}(\mathbf{r})=V_{B} \theta(R-\rho)$ with $V_{B} \rightarrow \infty(R=D / 2$ and $\rho$ is the transverse coordinate from the cylindrical set $\rho, \varphi, z) . \hat{\Delta}$ in Eqs. (1a) and (1b) is related to the order parameter by $\hat{\Delta}=\Delta(\hat{\mathbf{r}})$. The asterisk in Eqs. (1a) and (1b) means the complex conjugate.

As a mean-field approach, the BdG equations are solved in a self-consistent manner, taking account of the selfconsistency relation

$$
\Delta(\mathbf{r})=g \sum_{i}\left\langle\mathbf{r} \mid u_{i}\right\rangle\left\langle v_{i} \mid \mathbf{r}\right\rangle\left[1-2 f_{i}\right],
$$

where $g>0$ is the coupling constant and $f_{i}=1 /\left(e^{\beta E_{i}}+1\right)$ is the Fermi distribution for the bogolons. The sum in Eq. (3) runs over the states with the single-electron energy $(q$ $\left.=\left|m_{e} \mathbf{v}_{s} / \hbar\right|\right)$

$$
\xi_{i}=\left[\left\langle u_{i}\left|\hat{H}_{e}\right|_{q=0} \mid u_{i}\right\rangle+\left\langle v_{i}\left|\hat{H}_{e}^{*}\right|_{q=0} \mid v_{i}\right\rangle\right] \in\left[-\hbar \omega_{D}, \hbar \omega_{D}\right],
$$

with $\omega_{D}$ the Debye frequency. Equation (4) introduces the cutoff related to the momentum $\mathbf{p}$, to remedy the well-known ultraviolet divergence in Eq. (3). In addition, notice that there are two branches of solutions of the $\mathrm{BdG}$ equations $(i,+)$ and $(i,-)$ (see Refs. 24 and 25) with $\left.E_{i,+}\right|_{q=0}>0$ and $\left.E_{i,-}\right|_{q=0}$ $<0$. The sum in Eq. (3) should be taken over the physical states [the $(i,+)$ branch], i.e., $E_{i}=E_{i,+}$. Notice that this choice leads to the same results as the Gor'kov equations. ${ }^{25}$ It is of importance to remark that some of $E_{i}$ can become negative at $q \neq 0\left(v_{s} \neq 0\right)$. This is a signature of a reconstruction of the ground state due to the current-induced depairing of electrons. Indeed, the usual expression for the ground-state energy in the Bogoliubov theory (neglecting the term $\mu N_{e}$ with $N_{e}$ the number of electrons) is given by ${ }^{25,26}$

$$
E=\int \mathrm{d}^{3} r \frac{|\Delta(\mathbf{r})|^{2}}{g}-2 \sum_{i} E_{i}\left\langle v_{i} \mid v_{i}\right\rangle .
$$

When bogolons with negative energies appear, they survive at zero temperature and, so, give rise to corrections to the ground-state energy, i.e., $E \rightarrow E+\Delta E$ with

$$
\Delta E=2 \sum_{i, E_{i}<0} E_{i},
$$

a feature typical for the gapless superconductivity. ${ }^{24-26}$ 
For a given mean electron density $n_{e}=N_{e} /\left(\pi R^{2} L\right)$ the chemical potential $\mu$ is determined from

$$
n_{e}=\frac{2}{\pi R^{2} L} \sum_{i}\left[\left\langle u_{i} \mid u_{i}\right\rangle f_{i}+\left\langle v_{i} \mid v_{i}\right\rangle\left(1-f_{i}\right)\right] .
$$

Notice that the sum in Eq. (7) is convergent, and, so, the restriction to the Debye window [see Eq. (4)] is not needed here. For conventional superconductors the energy gap is typically much smaller than the chemical potential. As a result, $\mu$ stays practically the same when passing from the normal state to the superconducting one. ${ }^{24,27}$ So, it is easier to solve Eq. (7) in the absence of the superconducting order [for $\Delta(\mathbf{r})=0$ ], before the main procedure of numerically investigating a superconducting solution of the $\mathrm{BdG}$ equations. The chemical potential approaches the Fermi level $E_{F}$ when $T \rightarrow 0$ (which is not true for nanograins). As discussed in our previous papers, ${ }^{18}$ when working with the $\mathrm{BdG}$ equations in the parabolic band approximation, one needs to introduce an effective Fermi level, in order to obtain the correct results for the quantum-size superconducting oscillations. For aluminum (below we limit ourselves to this superconducting material) $E_{F}=0.9 \mathrm{eV}$ can be chosen when $D \gg 1-2 \mathrm{~nm}$ (see the discussion in Ref. 18 and comparison with the experimental results in Ref. 20). For extremely thin nanowires $(D$ $\sim 1 \mathrm{~nm}) E_{F}$ (and $\mu$, as well) will be systematically shifted up, as follows from Eq. (7).

Below it is natural to restrict ourselves to the situation when a superflow is parallel to the nanowire, $\mathbf{v}_{s}=\left(0,0, v_{s}\right)$. In this case the order parameter depends only on the transverse coordinate, i.e., $\Delta(\mathbf{r})=\Delta(\rho)$, and we arrive at

$$
\left\langle\mathbf{r} \mid \Psi_{i}\right\rangle=\frac{e^{\imath m \varphi}}{\sqrt{2 \pi}} \frac{e^{\imath k z}}{\sqrt{L}}\left(\begin{array}{c}
u_{j m k}(\rho) \\
v_{j m k}(\rho)
\end{array}\right),
$$

where $i=\{j, m, k\}$, with $j$ the radial quantum number, $m$ is the azimuthal quantum number, and $k$ is the wave vector of the quasi-free-electron motion parallel to the nanowire. Along the $z$ direction we use periodic boundary conditions with a unit cell of length $L$. In the transverse direction the quantumconfinement boundary conditions

$$
\left.u_{j m k}(\rho)\right|_{\rho=R}=\left.v_{j m k}(\rho)\right|_{\rho=R}=0
$$

should be imposed. The order parameter $\Delta(\rho)$ can be taken real together with $u_{j m k}(\rho)$ and $v_{j m k}(\rho)$ in our scheme. ${ }^{25,26}$ For the laboratory framework one needs to introduce position-dependent phase shifts, i.e., $\Delta \rightarrow \Delta e^{i 2 q z}, u$ $\rightarrow u e^{l q z}, v \rightarrow v e^{-l q z}$ (see Ref. 24). Details of the method used in the present work to numerically solve the Bogoliubov-de Gennes equations, are given in Appendix A.

In the presence of the superfluid motion of electrons, it is of interest to study how the supercurrent depends on the superfluid velocity $v_{s}$. The mean supercurrent density is given by

$$
\mathbf{j}_{s}=\frac{2 e}{m_{e} \pi R^{2} L} \sum_{i}\left[\left\langle u_{i}|\hat{\mathbf{P}}| u_{i}\right\rangle f_{i}+\left\langle v_{i}\left|\hat{\mathbf{P}}^{*}\right| v_{i}\right\rangle\left(1-f_{i}\right)\right],
$$

with $\hat{\mathbf{P}}^{*}=-\hat{\mathbf{p}}+m_{e} \mathbf{v}_{s}$. Similar to Eq. (7), the sum in Eq. (10) is over physical states [the $(i,+)$ branch of the solution] and perfectly convergent. Using Eq. (8) and the cylindricalcoordinate representation

$$
\nabla=\mathbf{e}_{\rho} \partial_{\rho}+\frac{\mathbf{e}_{\varphi}}{\rho} \partial_{\varphi}+\mathbf{e}_{z} \partial_{z}
$$

with $\mathbf{e}_{\rho}, \mathbf{e}_{\varphi}$, and $\mathbf{e}_{z}$ the corresponding unity vectors, we can rewrite Eq. (10) as $\left[\mathbf{j}_{s}=\left(0,0,-j_{s}\right)\right]$

$$
\begin{aligned}
j_{s}= & \frac{|e|}{\pi^{2} R^{2}} \sum_{j m} \int_{-\infty}^{+\infty} \mathrm{d} k\left[\frac{\hbar(k+q)}{m_{e}} \mathcal{U}_{j m k}^{2} f_{j m k}\right. \\
& \left.-\frac{\hbar(k-q)}{m_{e}} \mathcal{V}_{j m k}^{2}\left(1-f_{j m k}\right)\right],
\end{aligned}
$$

where it is convenient (see Appendix B) to introduce the real quantities $\mathcal{U}_{j m k}$ and $\mathcal{V}_{j m k}$,

$$
\begin{aligned}
& \mathcal{U}_{j m k}^{2}=\left\langle u_{j m k} \mid u_{j m k}\right\rangle=\int_{0}^{R} \mathrm{~d} \rho \rho u_{j m k}^{2}(\rho), \\
& \mathcal{V}_{j m k}^{2}=\left\langle v_{j m k} \mid v_{j m k}\right\rangle=\int_{0}^{R} \mathrm{~d} \rho \rho v_{j m k}^{2}(\rho),
\end{aligned}
$$

with the usual constraint ${ }^{24}$

$$
\mathcal{U}_{j m k}^{2}+\mathcal{V}_{j m k}^{2}=1 \text {. }
$$

We remark that in the presence of the longitudinal supercurrent, $f_{j m k} \neq f_{j, m,-k}$ (see Appendix B), and, so, the terms proportional to $k$ should be kept in Eq. (12).

It is instructive to present another expression for the supercurrent density (see Appendix C), namely,

$$
j_{s}=|e| n_{e} v_{s}-j_{n}=|e| n_{s} v_{s},
$$

with $j_{n}$ the contribution of the normal component and $n_{s}$ the density of the superfluid component. According to Eq. (15), the supercurrent is the current of all electrons moving with the superfluid velocity $v_{s}$ minus the term due to the normal component (bogolons). The minus sign appears because a normal current is dissipative in equilibrium, and, so, the normal component cannot participate in the superfluid motion.

\section{QUANTUM-SIZE CASCADES}

In this section we investigate how the superconducting condensate is suppressed by the longitudinal supercurrent in the presence of a quantized spectrum of the transverse electron motion. In this case the band of single-electron states splits up into a series of subbands. When the nanowire diameter increases, these subbands move down in energy. Each time when the bottom of a subband passes through the Fermi surface, the density of single-electron states at the Fermi level increases abruptly, which results in a sequence of width-dependent peaks. As a consequence, any superconducting quantity exhibits quantum-size oscillations with remarkable resonant enhancements. ${ }^{13}$ In addition, the superconducting order parameter is position dependent in the presence of quantum confinement. Therefore, one can expect that transverse quantization of the electron motion can have a 

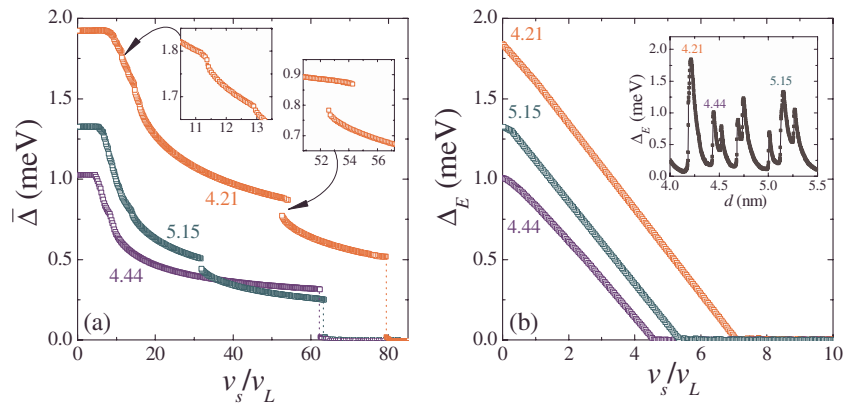

FIG. 1. (Color online) (a) The spatially averaged order parameter $\bar{\Delta}$ and (b) the energy gap $\Delta_{E}$ versus the superfluid velocity (in units of the bulk Landau velocity $v_{L}$ ) at zero temperature for three resonant diameters $d=4.21,4.44$, and $5.15 \mathrm{~nm}$. The inset in (b) shows quantum-size oscillations of the energy gap as a function of the wire diameter $d$.

strong impact on the superconductor-to-normal transition driven by the longitudinal supercurrent. Notice that the present investigation supplements our recent work about magnetically induced suppression of superconductivity in metallic nanowires. ${ }^{20}$

Numerical calculations were performed with the set of parameters typical of aluminum: ${ }^{24,27} \hbar \omega_{D}=32.31 \mathrm{meV}$, $g N(0)=0.18$, with $N(0)$ the bulk density of states at the Fermi level. The BCS coherence length for the chosen parameters is $\xi_{0}=1.6 \mu \mathrm{m}$, which is much larger than the nanowire diameters considered in the present work. The length of the unit cell in the longitudinal direction was taken $L$ $=5 \mu \mathrm{m} \gg \lambda_{F}=2 \pi / k_{F}$.

Figures 1(a) and 1(b) display how the spatially averaged order parameter $\bar{\Delta}$ and the energy gap $\Delta_{E}$ depend on the superfluid velocity for three resonant diameters $d=4.21$, 4.44, and $5.15 \mathrm{~nm}$ (at $T=0$ ). The superfluid velocity $v_{s}$ is here shown in units of the Landau (bulk) critical velocity, ${ }^{24-26}$ i.e., $v_{L}=\Delta_{\text {bulk }} / \hbar k_{F}$ (for the chosen parameters $\Delta_{\text {bulk }}=0.25 \mathrm{meV}$ and $\left.v_{L}=\Delta_{\text {bulk }} / \hbar k_{F}=78.1 \mathrm{~m} / \mathrm{sec}\right)$. Notice that the energy gap $\Delta_{E}$ is defined as the minimal nonnegative quasiparticle energy, and, so, in the presence of a sector of negative $E_{j m k}$ we get $\Delta_{E}=0$ (the gapless regime). The quantum-size oscillations of $\Delta_{E}$ as a function of the wire diameter at $T=0$ are given in the inset of Fig. 1(b). From Fig. 1(a) one can see that the destruction of the superconducting state occurs as a cascade of jumps in the superconducting order parameter. Some of these jumps are almost insignificant [see the left-hand-side inset in (a)]. Any jump is accompanied by clear signatures of the hysteretic behavior; see, for instance, the right-hand-side inset in (a), where details of the jump at $v_{s}=54.2 v_{L}(d=4.21 \mathrm{~nm})$ are presented.

To outline the physics behind these cascades of jumps in $\bar{\Delta}$, we need to learn more detail about the quasiparticle energies and the energy gap $\Delta_{E}$. As seen from Fig. 1(b), $\Delta_{E}$ behaves differently from $\bar{\Delta}$. First, $\Delta_{E}$ goes to zero much faster and in a large region of superfluid velocities it is zero with a nonzero pair condensate, which is related to the gapless regime. Second, there are no jumps in $\Delta_{E}$, and third, the energy-gap dependence on the superfluid velocity has a poliline structure. All these features can easily be understood

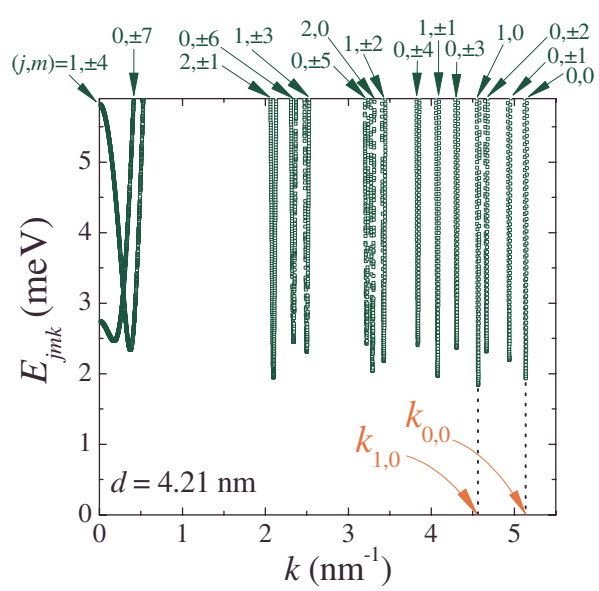

FIG. 2. (Color online) Quasiparticle energies $E_{j m k}$ versus $k$ (only $k>0$ are given) for $d=4.21 \mathrm{~nm}$ at $v_{s}=0$.

with the help of Anderson's approximation (see Appendix B) that results in $E_{j m k}$ given by Eq. (B7). Due to the Doppler term $E_{\text {Dopp }}=\hbar^{2} \mathrm{kq} / \mathrm{m}$, the quasiparticle energies with $k<0$ (with $k>0$ ) shift down (up) with increasing the superfluid velocity, and such shifts are linear in $v_{s}$. Furthermore, since the relevant states of each single-electron subband (the states situated in the Debye window) occupy only two narrow region in 1D $k$ space, we can say that, to a certain approximation, each quasiparticle branch can be specified by two Doppler-shift terms: $E_{\text {Dopp }, j m}^{(+)}=\hbar k_{j m} v_{s}$ for $k>0$, and $E_{\text {Dopp }, j m}^{(-)}$ $=-\hbar k_{j m} v_{s}$ for $k<0$, with $k_{j m}=\sqrt{2 m\left(\mu-\xi_{j m k}\right)}$. This approximation is quite accurate for excitations in the single-electron subbands with the bottoms far below the Fermi level. In Fig. $2 E_{j m k}$ are shown versus $k$ (at $k>0$ ) for $d=4.21 \mathrm{~nm}$ and $v_{s}$ $=0$. As seen, for the energy gap we have $\Delta_{E}=\Delta_{1,0}$. Notice that $\Delta_{j m}$ is defined by Eq. (B1), and, at $v_{s}=0$, it is the energy gap in the corresponding single-electron subband [see Eq. (B7)]. For $v_{s}>0$ the subband-dependent energy gap is no longer $\Delta_{j m}$ but $\Delta_{j m}-\hbar v_{s} k_{j m}$ instead. As follows from Fig. 2, one can expect that at small enough values of the superfluid velocity we have $\Delta_{E}=\Delta_{1,0}-\hbar v_{s} k_{1,0}$. Now, let us take into account that at zero temperature $\Delta_{j m}$ does not change with $v_{s}$ for $\Delta_{E}>0$ [see, for instance, Eq. (B9) with $1-2 f_{j m k}=1$ in this case]. Hence, $\Delta_{E}$ can have only a poliline structure, and for small enough values of $v_{s}$ its slope is $\hbar k_{1,0}$. However, as seen from Fig. $2, k_{1,0}<k_{0,0}$ while $\Delta_{1,0}$ is nearly the same as $\Delta_{0,0}$. So, at a certain value of the superfluid velocity, a kink can be expected in $\Delta_{E}$ as a function of $v_{s}$, when its slope changes from $\hbar k_{1,0}$ to $\hbar k_{0,0}$. This weak kink does occur at $v_{s} \approx v_{L}$, as seen from Fig. 1(b). We remark that for $T>0$ such a kink is smeared out because $\Delta_{j m}$ becomes sensitive to the superfluid velocity even for $\Delta_{E}>0\left[1-2 f_{j m k} \neq 1\right.$ when $T$ $>0]$.

Now let us return to the cascades of jumps in the spatially averaged superconducting condensate $\bar{\Delta}$. The contribution of any single-electron subband to the superconducting order parameter depends strongly on the fact whether or not there are excitations with negative energies in this subband [see the self-consistency relation given by Eq. (3)]. Hence, when analyzing the pronounced jumps in $\bar{\Delta}$, it seems promising to consider the quasiparticle energies of the most important 

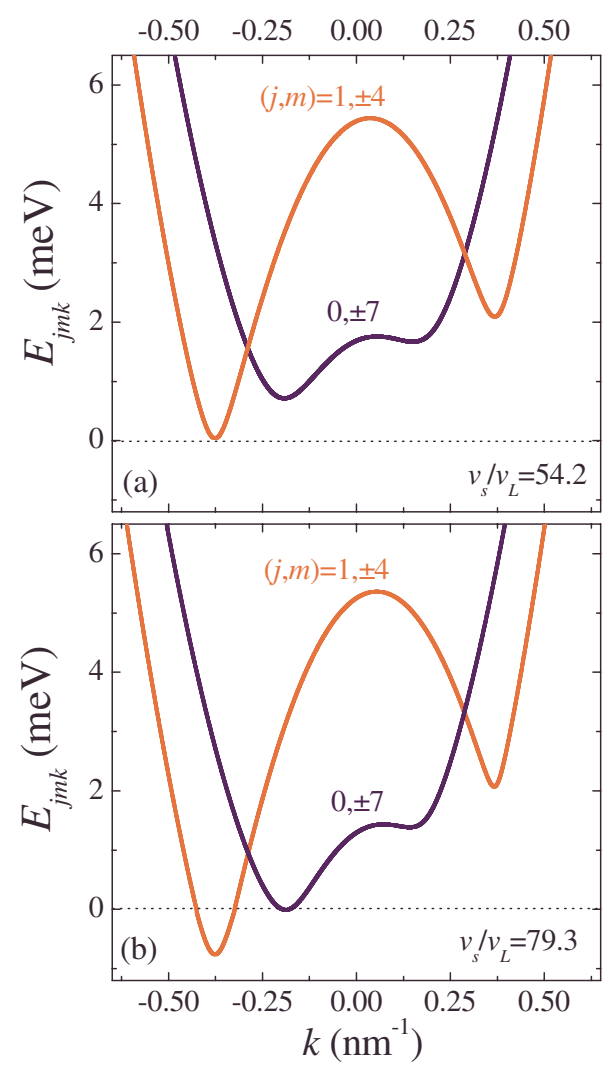

FIG. 3. (Color online) Dispersion curves for the resonant branches $j=0, m= \pm 7$ and $j=1, m= \pm 4$ at $d=4.21 \mathrm{~nm}$ : (a) $v_{s} / v_{L}$ $=54.2$ and $(\mathrm{b}) v_{s} / v_{L}=79.3$.

single-electron subbands making the major contribution to the superconducting characteristics. In particular, for the resonant diameter $d=4.21 \mathrm{~nm}$, there are four such singleelectron subbands, i.e., $(j, m)=(0, \pm 7),(1, \pm 4)$. In Fig. 3 the dispersion curves for the corresponding excitations are shown for two cases: (a) $v_{s}=54.2 v_{L}$ (the first profound jump in $\bar{\Delta}$ ) and (b) $v_{s}=79.3 v_{L}$ (the second significant drop). As seen, in the presence of a longitudinal supercurrent, the dispersion curves for both quasiparticle branches are tilted with respect to $k=0$, in total agreement with Eq. (B7). Figures 3(a) and 3(b) illustrate the fact that each time when a quasiparticle branch touches zero, a discontinuity (jump) in the spatially averaged order parameter occurs. A profound jump in $\bar{\Delta}$ arises when quasiparticles with negative energies appear in one of the single-electron subbands that is responsible for a major contribution to the order parameter. Shifts to negative energies of other bogolons can produce only insignificant jumps or even kinks in $\bar{\Delta}\left(v_{s}\right)$. Quasiparticles with negative energies are a signature of the destruction of the Cooper pairs (see Ref. 25 and discussion in Ref. 20). Thus, the supercurrent-induced depairing of electrons is the reason for the cascades of jumps in $\bar{\Delta}$ as a function of the superfluid velocity. In the presence of the transverse quantization such a depairing is not uniform but subband dependent. In this case the decay of the order parameter driven by the supercurrent is accompanied by a nonuniform depletion of contributions from different transverse modes. Hence, in addition to a de-
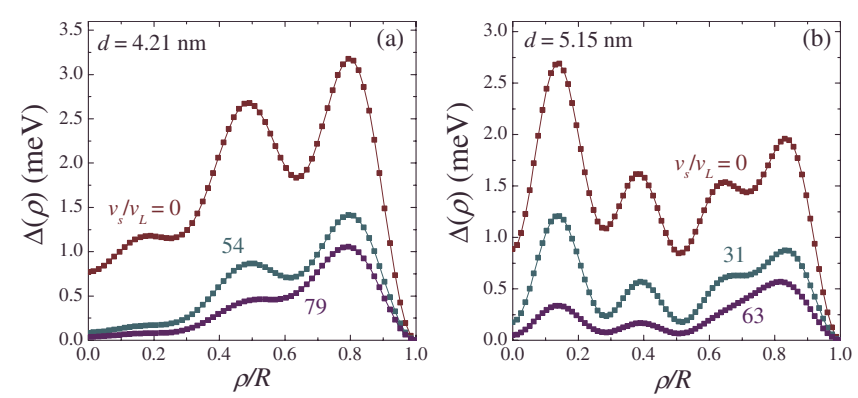

FIG. 4. (Color online) The transverse distribution of the pair condensate $\Delta(\rho)$ for (a) $d=4.21 \mathrm{~nm}\left[\right.$ at $\left.v_{s}=0,54 v_{L}, 79 v_{L}\right]$ and (b) $d=5.15 \mathrm{~nm}\left[\right.$ at $\left.v_{s}=0,31 v_{L}, 63 v_{L}\right]$.

crease in the spatially averaged value of $\Delta(\rho)$, a change in its profile can be expected when increasing $v_{s}$. This is clearly seen in Fig. 4, where the $v_{s}$-dependent evolution of $\Delta(\rho)$ is shown for (a) $d=4.21 \mathrm{~nm}$ and (b) $d=5.15 \mathrm{~nm}$ (for $T=0$ ).

The spatially averaged condensate $\bar{\Delta}$ for these diameters is given in Fig. 1. Notice that for both situations there exist two jumps: at $v_{s} / v_{L}=54.2$ and 79.3 for $d=4.21 \mathrm{~nm}$, and at $v_{s} / v_{L}=31.7$ and 63.5 for $d=5.15 \mathrm{~nm}$. In Fig. 4(a) $\Delta(\rho)$ is shown for $d=4.21 \mathrm{~nm}$ for different values of $v_{s}$. In addition to $v_{s}=0$, we show the results for $v_{s} / v_{L}=54$ (just before the first jump) and 79 (the vicinity of the final jump down to zero). As seen, the profile of $\Delta(\rho)$ changes significantly. In particular, for $v_{s}=0$ there are two pronounced maxima in $\Delta(\rho)$, i.e., at $\rho / R=0.47$ and $\rho / R=0.8$. For $v_{s} / v_{L}=79$, the maximum at $\rho / R=0.47$ is completely washed out. The data for $d=5.15 \mathrm{~nm}$ are presented in Fig. 4(b) for $v_{s} / v_{L}=0,31$ (just before the first jump) and 63 (near the eventual jump to zero). It is clear that the profile of $\Delta(\rho)$ at $v_{s} / v_{L}=63$ is completely different as compared to $v_{s}=0$.

We remark that the resonant single-electron subbands have much smaller Doppler shifts since their bottoms, being situated in the Debye window, are close to the Fermi level. This is why pronounced jumps in $\bar{\Delta}\left(v_{s}\right)$ appear for large enough superfluid velocities and accompany the total decay of the superconducting condensate. Notice that the singleelectron subbands with large $k_{j m}$ can be more sensitive to surface roughness and imperfections. Consequently, the kinks and small jumps in $\bar{\Delta}$ calculated for the case of a uniform cross section would be smeared out in the presence of imperfections. The pronounced jumps can be expected to be more stable. In general, the quantum-size cascades induced by a longitudinal supercurrent look similar to the cascades in $\bar{\Delta}$ induced by a magnetic field parallel to a nanowire. ${ }^{20}$ In both situations the quantum-size cascades survive up to $d=10-15 \mathrm{~nm}$, being washed out for larger diameters (see Fig. 5). For both the longitudinal supercurrent and parallel magnetic field, the cascade structure is not only typical for the resonant diameters but it is also found for the off-resonant diameters [the points where the thicknessdependent superconducting characteristics drop below their bulk values, see the inset in Fig. 1(b)]. To illustrate this fact, we show in Fig. $6 \bar{\Delta}$ for off-resonant diameters $d=5.5,7.6$, and $10.85 \mathrm{~nm}$. Note that the off-resonant drops in $\bar{\Delta}$ are reduced when $d$ increases. It is also of importance to under- 


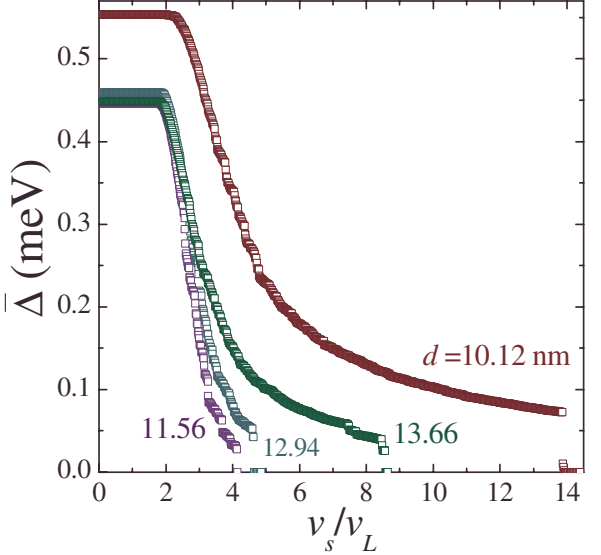

FIG. 5. (Color online) The spatially averaged condensate $\bar{\Delta}$ as a function of $v_{s} / v_{L}$ for the resonant diameters $d=10.12,11.56,12.94$, and $13.66 \mathrm{~nm}$.

line differences of the present cascades with respect to those in a paramagnetic field. For the setup with a parallel magnetic field, the superconducting condensate is "eaten" by the screening currents induced in the azimuthal direction. In this case the condensate is stable in the center of the nanowire, and the transverse modes with $m=0$ cannot be directly influenced by the magnetic field. ${ }^{20}$ As a result, there are diameters for which the superconducting state cannot be destroyed by the orbital effects without invoking the Pauli paramagnetism. In the present case there is no preference for any point of the nanowire cross section (the superfluid velocity does not depend on the transverse and azimuthal coordinates). As a consequence, we do not observe any superconducting resonances that are extremely stable. The appearance of the cascade structure in the presence of the longitudinal supercurrent makes it possible to expect that similar cascades can exist for superconducting nanowires in perpendicular magnetic fields. If so, the ratio of the parallel to perpendicular critical magnetic field can significantly deviate from the usual mesoscopic regime due to possible quantum-size oscillations.

It is necessary to stress that smoothing of the quantumsize cascades for $d \sim 20 \mathrm{~nm}$ does not mean that we immedi-

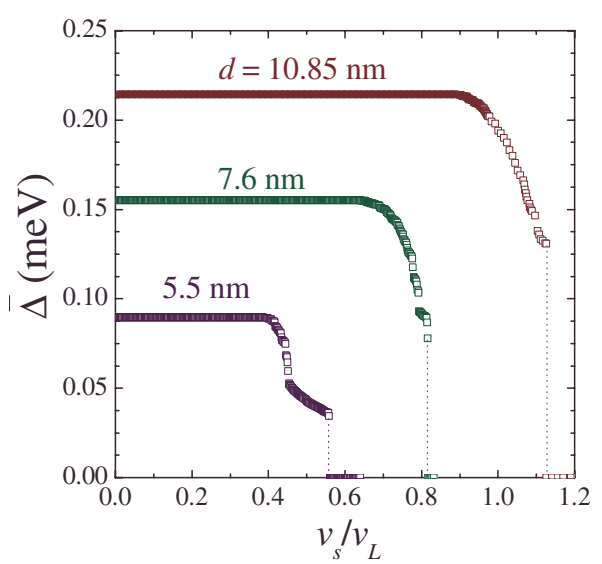

FIG. 6. (Color online) $\bar{\Delta}$ as a function of the relative superfluid velocity $v_{s} / v_{L}$ for the off-resonant diameters $d=5.5,7.6$, and 10.85 nm.

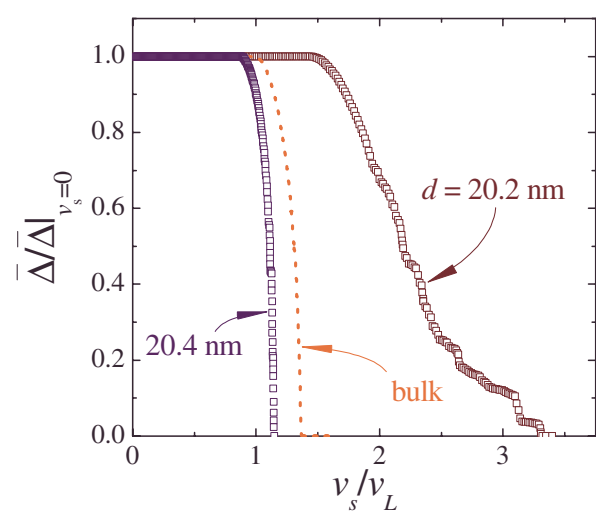

FIG. 7. (Color online) $\bar{\Delta}$ versus $v_{s} / v_{L}$ for the resonant diameter $d=20.2 \mathrm{~nm}$ and off-resonant diameter $d=20.4 \mathrm{~nm}$. The dotted curve represents the bulk results.

ately arrive at the bulk regime. In Fig. 7 the ratio $\bar{\Delta} /\left.\bar{\Delta}\right|_{v_{s}=0}$ is shown as a function of $v_{s}$ for the resonant and off-resonant diameters, $d=20.2 \mathrm{~nm}$ and $d=20.4 \mathrm{~nm}$, respectively (together with the bulk result given by the dotted curve). While the off-resonant points are close to the bulk dependence, the resonant data still exhibit significant deviations from the bulk curve. Though jumps are washed out, the cascade structure of continuous steplike drops is still seen. The same is valid for any resonant point when $d \sim 20-40 \mathrm{~nm}$. The bulk regime is obtained only when the superconducting resonances are completely decayed (for $d \approx 50-70 \mathrm{~nm}$ ).

We would like to emphasize that the above discussed results are for $T=0$. At finite temperatures the situation changes. As seen from Fig. 8 all the kinks and small jumps are washed out rapidly even at very low temperatures. The first pronounced jump at $v_{s}=54.2 v_{L}$ survives up to $T=1 \mathrm{~K}$ $\left(T / T_{c}=0.08\right.$ with $T_{c}$ the critical temperature at $\left.v_{s}=0\right)$ and then degenerates into a smooth steplike drop. As for the jump to zero at $v_{s}=79.3 v_{L}$, it remains up to $T=4 \mathrm{~K}\left(T / T_{c}=0.32\right)$. However, its position shifts to smaller values of $v_{s}$. There are no signatures of jumps for $T / T_{c}>0.32$, and $\bar{\Delta}$ smoothly goes to zero with increasing $v_{s}$.

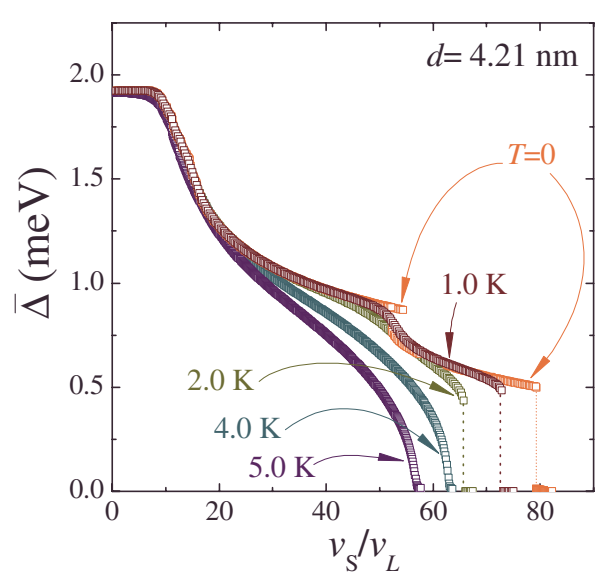

FIG. 8. (Color online) The spatially averaged condensate $\bar{\Delta}$ versus $v_{s} / v_{L}$ for $d=4.21 \mathrm{~nm}$ at $T=0,1.0,2.0,4.0$, and $5.0 \mathrm{~K}$. 


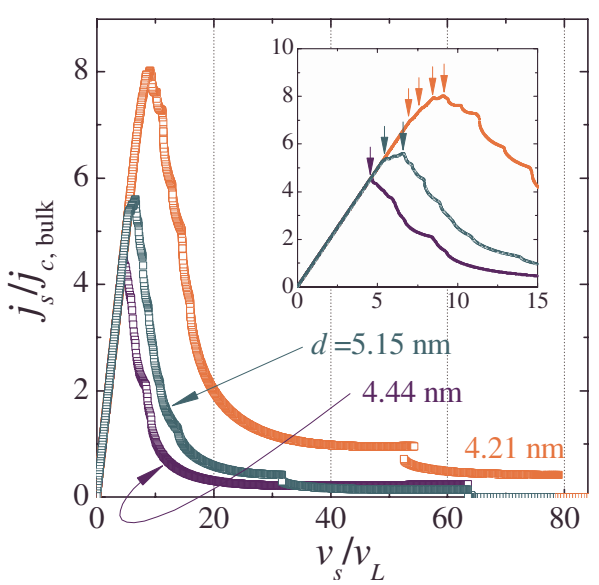

FIG. 9. (Color online) The supercurrent density $j_{s}$ in units of the bulk critical current density $j_{c \text {, bulk }}$ versus the relative superfluid velocity $v_{s} / v_{L}$ for $d=4.21,4.44$, and $5.15 \mathrm{~nm}$ at $T=0$.

\section{CRITICAL CURRENT AND SUPERFLUID DENSITY}

In this section we investigate how the transverse quantization can influence the supercurrent density $j_{s}$ (together with the related critical current $j_{c}$ and superfluid density $n_{s}$ ). Numerical results for $j_{s}$ as function of the superfluid velocity at $T=0$ are shown in Fig. 9 for three resonant diameters $d$ $=4.21,4.44$ and $5.15 \mathrm{~nm}$. As expected, at small values of $v_{s}$ there is a linear increase in the current density with the superfluid velocity. This is typical of the gap regime $\left(\Delta_{E} \neq 0\right)$, when there are no excitations in the system (at $T=0)$ and so, $j_{n}=0$ and $j_{s}=|e| n_{e} v_{s}$ [see, for details, Eq. (15) and Appendix C]. At a certain superfluid velocity, the first quasiparticle branch touches zero and so, the energy gap disappears, i.e., $\Delta_{E}=0$. This velocity can be referred to as the first depairing velocity $v_{s, 1}$ being the analog of the Landau depairing velocity $v_{L}=\Delta_{\text {bulk }} / \hbar k_{F}$ in bulk. With the subsequent increase in $v_{s}$, the supercurrent density $j_{s}$ exhibits a sequence of kinks (they sometimes are accompanied by small jumps), shown in the inset of Fig. 9 by arrows. Similar to the dependence of $\bar{\Delta}$ on $v_{s}$, a kink (or small jump) in $j_{s}$ as a function of $v_{s}$ occurs each time when excitations with negative energies appear in a new single-electron subband making a minor contribution to the superconducting characteristics. At $v_{s}>v_{s, 1}$ the current density $j_{s}$ usually continues to exhibit an overall increase with $v_{s}$ (see, for instance, the results for $d=4.21 \mathrm{~nm}$ and $5.15 \mathrm{~nm}$ in Fig. 9) until the maximum value $j_{c}$ (the widthdependent critical current density) is reached. Notice that sometimes $j_{c}$ is already reached at $v_{s}=v_{s, 1}$, e.g., see the data for $d=4.44 \mathrm{~nm}$ in Fig. 9. A strong decay of the supercurrent density follows immediately after reaching the maximum. However, the interval of such a decay is seen to be narrow. Subsequently, an extended region is found where $j_{s}$ rather slowly varies with $v_{s}$ before a complete suppression of $j_{s}$ occurs at the second depairing velocity $v_{s, 2}$, which can be treated as the critical velocity $v_{c} \equiv v_{s, 2}$. At this point bogolons with negative energies appear in the single-electron subband responsible for the resonant enhancement, and the order parameter immediately drops down to zero. If two (or more) single-electron subbands give a major contribution to the ba-

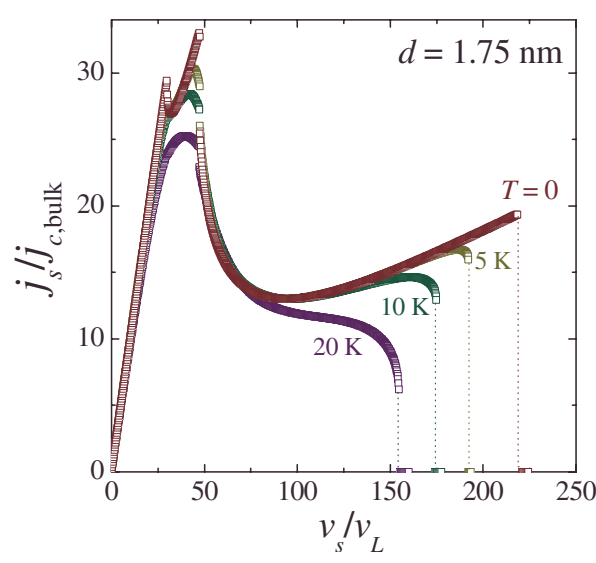

FIG. 10. (Color online) The relative supercurrent density $j_{s} / j_{c \text {, bulk }}$ versus $v_{s} / v_{L}$ for $d=1.75 \mathrm{~nm}$ (the resonant diameter) at $T$ $=0,5,10$, and $20 \mathrm{~K}$.

sic superconducting characteristics, there exist two (or more) pronounced jumps in $\bar{\Delta}$ and $j_{s}$ as function of the superfluid velocity (see $d=4.21$ and $5.15 \mathrm{~nm}$ in Fig. 9). It is of importance that $j_{s}$ exhibits a linear increase with $v_{s}$ in the vicinity of any pronounced jump (recall that the superconducting state is unstable when $j_{s}$ decreases with $v_{s}$ ). However, the slope of such an increase is not $|e| n_{e}$ any more, like it was for $v_{s} \rightarrow 0$ but $|e| n_{s}$ with $n_{s} \ll n_{e}$. Increasing $T$ smooths out all jumps and shortens the regions, where the current density increases, as seen from Fig. 10, where $j_{s}$ as a function of $v_{s} / v_{L}$ is plotted versus the four temperatures $T=0,5,10$, and $20 \mathrm{~K}$ [for the resonant diameter $d=1.75 \mathrm{~nm}$ ]. In the offresonance regime most of the kinks and jumps in $j_{s}$ as a function of $v_{s}$ disappear but the eventual drop to zero survives at $v_{s}=v_{c}$, as seen from Fig. 6 .

The physics behind the strong decay of the current density after reaching the critical current density $j_{c}$ is the appearance of quasiparticles with negative energies. Excitations cannot participate in a nondissipative flow and so, it should be reduced due to depletion of the pair superconducting condensate. For superfluid velocities much smaller than $v_{c}$, the density of excitations is almost insignificant. Therefore, it is intriguing why their effect is so pronounced, resulting in almost an abrupt drop of the supercurrent density just after $v_{s}=v_{s, 1}$. The reason is that quasiparticles with negative energies, which appear in the vicinity of $v_{s, 1}$, have large longitudinal momenta of the order of $4-5 \mathrm{~nm}^{-1}$ (this is about 3D Fermi momentum $k_{F}$; for our effective Fermi level $E_{F}$ $=0.9 \mathrm{eV}$ we have $\left.k_{F}=4.86 \mathrm{~nm}^{-1}\right)$. See, for instance, Fig. 3 . It is instructive to compare $k_{F}$ with $\hbar v_{s, 1} / m_{e}$, which allows us to conclude that $k_{F}$ is larger by at least three to four orders of magnitude! So, even a small number of quasiparticles can produce a significant contribution to $j_{n}$ [see Eq. (C5)], which remarkably reduces $j_{s}$. From Fig. 11, where the supercurrent density $j_{s}$ is plotted versus $v_{s} / v_{L}$ together with $j_{n}$, one notices a rapid increase in $j_{n}$ taking place just in the vicinity of the first depairing velocity. It is of interest to compare $j_{s}$ $=|e| n_{s} v_{s}$ with $|e| n_{e} v_{s}$ given by the solid line in the insets. For small $v_{s}$, i.e., in the gap regime, $n_{s}=n_{e}$ and $j_{s}=|e| n_{e} v_{s}$. For the superfluid velocities close to $v_{c}$, we have $j_{n} \rightarrow|e| n_{e} v_{s}$ and, hence, a great suppression of the supercurrent density $j_{s}$. 

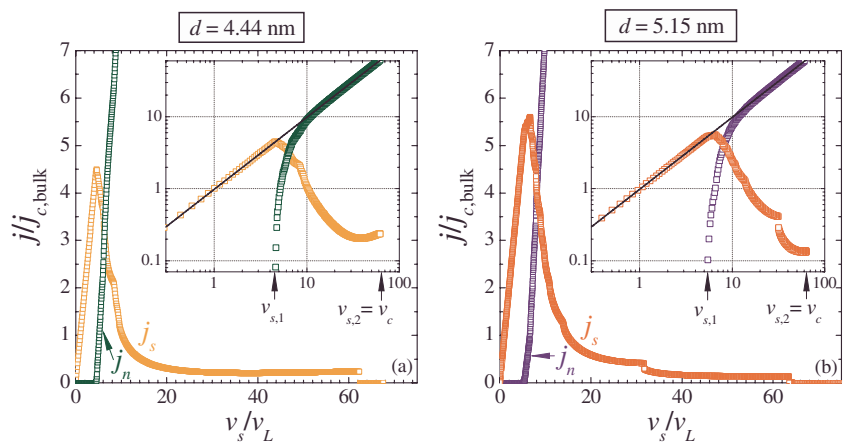

FIG. 11. (Color online) The supercurrent density $j_{s}$ together with the normal-component contribution $j_{n}$ versus $v_{s} / v_{L}$ [at $T=0$ ] for $d=4.44 \mathrm{~nm}$ (a) and $=5.15 \mathrm{~nm}$ (b). The insets in both panels show the same data but for larger current values and on a log-log scale, together with the solid line representing $|e| n_{e} v_{s}$.

Such a suppression is a result of a significant drop in the superfluid density $n_{s}$ from $n_{e}$ down to values of about $0.01-0.1 n_{e}$, as seen in Fig. 12, where $n_{s}$ is plotted in units of the electron density $n_{e}$ versus the relative superfluid velocity $v_{s} / v_{L}$ for the same diameters as in Fig. 9. In addition, $n_{s} / n_{e}$ calculated at the resonant diameter $d=1.75 \mathrm{~nm}$ is shown in the inset of Fig. 12, to illustrate how the nanowire diameter can influence the drop of $n_{s}$.

Figure 13 shows our numerical results for the longitudinal critical current density as a function of the nanowire diameter. Calculations were performed with steps of $\Delta d$ $=0.02 \mathrm{~nm}$. As can be expected, the formation of the transverse superconducting modes results in $j_{c}$ oscillating with the diameter. Such quantum-size oscillations are accompanied by resonant enhancements at which the supercurrent density increases significantly above its bulk value $j_{c \text {,bulk }}$ $\approx 1.03|e| n_{e} v_{L}{ }^{25,26}$ The off-resonant diameters exhibit drops of $j_{c}$, sometimes even below the bulk limit. However, the resonant enhancements are more pronounced. For instance, $\int_{4 \mathrm{~nm}}^{6 \mathrm{~nm}} j_{s} d d=1.88 \int_{4 \mathrm{~nm}}^{6} j_{s, \text { bulk }} d d$. Real samples have inevitable cross-section fluctuations that will smooth the quantum-size oscillations of the critical current. However, the main in-

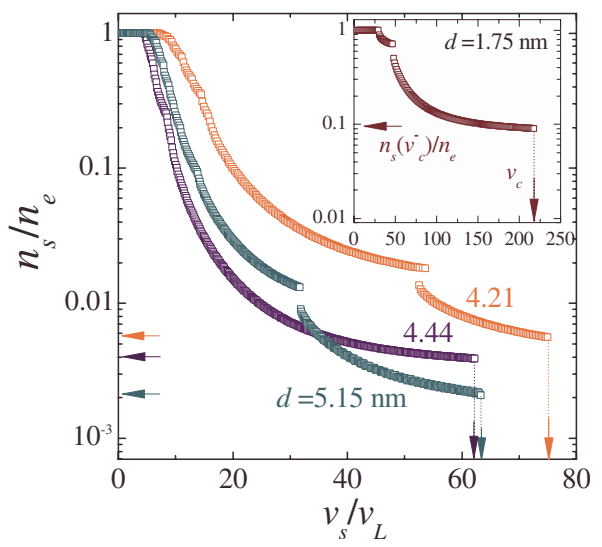

FIG. 12. (Color online) The relative superfluid density $n_{s} / n_{e}$ versus $v_{s} / v_{L}$ as calculated at zero temperature for the resonant diameters $d=4.21,4.44$, and $5.15 \mathrm{~nm}$. The inset shows the same but for $d=1.75 \mathrm{~nm}$, with $n_{s}\left(v_{c}^{-}\right)=\lim _{v_{s} \rightarrow v_{c}-0} n_{s}$. Arrows indicate critical velocities with the corresponding values of the superfluid density.

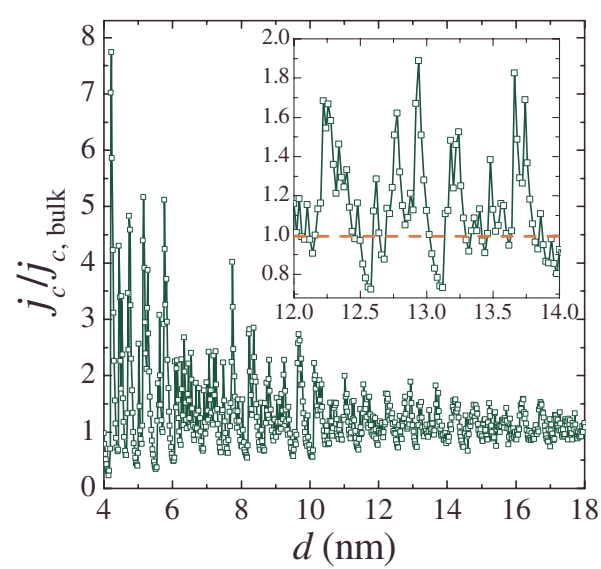

FIG. 13. (Color online) Quantum-size oscillations of the critical current density: $j_{c}$ given in units of the bulk critical current density $j_{c \text {,bulk }}$ versus the nanowire diameter at $T=0$ The inset shows an enlargement for diameters $d=12-14 \mathrm{~nm}$.

crease in the order parameter and, hence, of the critical current, comes from the resonant single-electron subband(s) whose bottom(s) are situated near the Fermi surface. Singleelectron states from such a subband are characterized by small values of $k$ and, consequently, they can be expected to be rather insensitive to the surface roughness and (nonmagnetic) imperfections. Therefore, the critical current at the resonant diameters will be stable with respect to inclusion of the roughness and impurity effects. At the off-resonant diameters, different subbands produce more or less the same contribution to the superconducting characteristics. These subbands have their bottoms significantly below the Fermi level and, hence, the relevant single-electron states have large (absolute) values of $k$, close to the 3D Fermi wave vector $k_{F}$. Such states are more sensitive to imperfections and, so, the off-resonant drops in the critical current would be smeared out by disorder. Thus, one can expect that disorder, i.e., nonmagnetic impurities and surface roughness, will smooth the quantum-size oscillation of the critical supercurrent into an overall increase with decreasing nanowire thickness.

\section{CONCLUDING REMARKS}

In conclusion, in this work we restricted ourselves to high-quality metallic superconducting nanowires, where the quantization of the transverse electron spectrum is not smeared by the scattering on nonmagnetic impurities. In this case spatial transverse variations in the pair condensate are of paramount importance. To take this important issue into account, we have employed the Bogoliubov-de Gennes wave-function formalism supplemented by Anderson's approximate semianalytical solution. Within this approach we have found that the superconductor-to-normal transition induced by a longitudinal supercurrent in a metallic cylindrical nanowire occurs as a cascade of jumps in the order parameter and supercurrent density as function of the superfluid velocity. Similar quantum-size cascades were recently calculated for a superconducting nanowire in a parallel magnetic field. ${ }^{20}$ We have also demonstrated that the temperature effects 
smooth out the jumps into steplike but continuous drops. The cascade structure was shown to be washed out for diameters $d>15-20 \mathrm{~nm}$. However, the bulk behavior is recovered only for much larger diameters $d=60-70 \mathrm{~nm}$, i.e., in the mesoscopic regime. We have shown that the critical current density exhibits quantum-size oscillations with pronounced resonant enhancements. Real samples exhibit inevitable cross-section fluctuations that will smooth the quantum-size oscillations, leading to an overall enhancement of the critical current density with decreasing nanowire thickness. Another consequence of such fluctuations is that they will smooth out jumps in the order parameter (and supercurrent) so that only the final drop down to zero can survive (compare results for the resonant and off-resonant diameters). It is of importance to note that the superconducting modes responsible for the resonant enhancements have long wavelengths in the longitudinal direction and, hence, are not very sensitive to disorder.

In the present manuscript we did not address the issue of phase fluctuations of the order parameter. For nanowires the fluctuation-induced phase slips can be of importance resulting in nonvanishing resistance down to low temperatures $T$ $\ll T_{c}$. However, for high-quality superconducting nanowires, such fluctuations were found to be significant only in extremely narrow samples with thickness less than 6-8 nm. For example, the sharp crossover from the superconducting state to the normal one due to fluctuations was observed in aluminum high-quality nanowires when the nanowire thickness reduces down to $8 \mathrm{~nm}$ (see the second paper in Ref. 4). However, no signature of such a crossover was observed in similar aluminum nanowires with the cross section of about $5.2 \mathrm{~nm} \times 6.1 \mathrm{~nm},{ }^{6}$ where the sample resistance due to the quantum-phase slippage was found to be less than $1 \%$ of the corresponding normal resistance at $T<0.9 T_{c}$. Thus, the quantum-cascade transitions in high-quality superconducting nanowires can be expected before the phase slippage destroys the superconducting state.

Another remark concerns the coupling constant $g$ that was taken in the present paper the same as in bulk. What about the well-known softening of phonons due to the surface effect? First, the concrete value of $g$ does not influence our results about quantum-size cascades and is only of importance for the thickness-dependent enhancement of the critical current density. Second, from a comprehensive study in paper, ${ }^{28}$ one can expect that for Al films, the phonon softening can result in an increase in the critical temperature by $\sim 10 \%$ at $d=5 \mathrm{~nm}$ as compared to bulk. From our previous investigations on nanofilms, ${ }^{18,29}$ we know that the quantumsize resonant enhancements of $T_{c}$ in Al nanofilms with thickness of $5 \mathrm{~nm}$ are about $50 \%$ (for $g$ the same as in bulk). From this we expect that phonon softening can significantly contribute to an additional enhancement of $j_{c}$ only for $d$ $<5 \mathrm{~nm}$.

Notice that probing the supercurrent in superconducting samples on a local scale has recently been addressed in Ref. 30. The developed technique based on scanning tunneling spectroscopy with a superconducting tip is highly sensitive to the Doppler-shift term in the superconductor quasiparticle spectrum of the sample, thus allowing the local study of the superfluid velocity. Therefore the investigation of the current-carrying state can be performed locally, and the supercurrent distribution in the nanostructures can be obtained experimentally.

\section{ACKNOWLEDGMENTS}

This work was supported by the Flemish Science Foundation (FWO-Vl), the Belgian Science Policy (IAP), and the ESF-AQDJJ and ESF-NES networks. One of the co-authors (M.D.C.) is grateful to the Academia Sinica (Taiwan).

\section{APPENDIX A: NUMERICAL SOLUTION OF THE BDG EQUATIONS}

To numerically solve the BdG equations, Eqs. (1a) and (1b) are converted into a matrix form by expanding in terms of the eigenfunctions of the single-electron Hamiltonian $\hat{H}_{e}$ [see Eq. (2)]. This form is numerically diagonalized, and iterations are invoked, to account for the self-consistency relation given by Eq. (3).

When inserting Eq. (8) into Eqs. (1a) and (1b), a system of two effectively 1D equations is derived for $u_{j m k}(\rho)$ and $v_{j m k}(\rho)$, the transverse parts of the particlelike and holelike wave functions, respectively. Then, $u_{j m k}(\rho)$ and $v_{j m k}(\rho)$ can be replaced by the series

$$
\left(\begin{array}{c}
u_{j m k}(\rho) \\
v_{j m k}(\rho)
\end{array}\right)=\sum_{J}\left(\begin{array}{l}
u_{j m k, J m} \\
v_{j m k, J m}
\end{array}\right) \vartheta_{J m}(\rho),
$$

with

$$
\vartheta_{J m}(\rho)=\frac{\sqrt{2}}{R \mathcal{J}_{m+1}\left(\alpha_{J m}\right)} \mathcal{J}_{m}\left(\alpha_{J m} \frac{\rho}{R}\right),
$$

where $\mathcal{J}_{m}(x)$ is the first-kind Bessel function of the $m$ th order, and $\alpha_{J m}$ is the $J$ node of this function. As a result, we arrive at the following system of matrix equations:

$$
\begin{aligned}
& {\left[E_{j m k}-T_{J m k}^{(+)}\right] u_{j m k, J m}=\sum_{J^{\prime}} \Delta_{J m, J^{\prime} m} v_{j m k, J^{\prime} m},} \\
& {\left[E_{j m k}+T_{J m k}^{(-)}\right] v_{j m k, J m}=\sum_{J^{\prime}} \Delta_{J m, J^{\prime} m} u_{j m k, J^{\prime} m},}
\end{aligned}
$$

where

$$
T_{J m k}^{( \pm)}=\frac{\hbar^{2}}{2 m_{e}}\left[\frac{\alpha_{J m}^{2}}{R^{2}}+(k \pm q)^{2}\right]-\mu
$$

and

$$
\Delta_{J m, J^{\prime} m}=\int_{0}^{R} \mathrm{~d} \rho \rho \vartheta_{J m}(\rho) \Delta(\rho) \vartheta_{J^{\prime} m}(\rho) .
$$

In addition, one should take into account the normalization condition $^{24}$

$$
\sum_{J}\left[u_{j m k, J m}^{2}+v_{j m k, J m}^{2}\right]=1,
$$

where $u_{j m k, J}$ and $v_{j m k, J}$ are real. 


\section{APPENDIX B: ANDERSON'S APPROXIMATION TO THE BDG EQUATIONS}

As described in Appendix $\mathrm{A}$, the $\mathrm{BdG}$ equations are solved by expanding the order parameter in the singleelectron wave functions. By this procedure we take into account the fact that, in general, the electronlike and holelike wave functions are not proportional to the single-electron wave function with the corresponding quantum numbers. However, Anderson's approximate solution, ${ }^{8}$ when seeking for the minimum of the free-energy functional in the subspace of $u_{j m k}(\rho)$ and $v_{j m k}(\rho)$ being proportional to $\vartheta_{j m}(\rho)$ [compare with Eq. (A1)], is very helpful for analyzing results obtained from a numerical solution of the $\mathrm{BdG}$ equations. Anderson's approximation is good enough provided that the pairing of two electrons from different single-electron subbands plays a minor role, i.e., $\Delta_{j m, j^{\prime} m} \simeq 0\left(j \neq j^{\prime}\right)$. This is true when the subband-dependent order parameter

$$
\Delta_{j m} \equiv \Delta_{j m, j m}
$$

is much less than the subband energy spacing $\delta_{s u b}$, i.e.,

$$
\Delta_{j m} \ll \delta_{s u b}=\frac{\hbar^{2}}{2 m_{e}} \frac{\pi^{2}}{R^{2}} .
$$

As seen, the smaller the nanowire width the better the approximation. However, we remark that this approximation becomes exact only in the situation of the spatially independent order parameter for which, as follows from Eq. (A5), $\Delta_{j m, j^{\prime} m}=0$ for $j \neq j^{\prime}$.

With this in mind, we outline Anderson's approximate solution to the $\mathrm{BdG}$ equations. The basic assumption is given by

$$
u_{j m k}(\rho)=\mathcal{U}_{j m k} \vartheta_{j m}(\rho), \quad u_{j m k}(\rho)=\mathcal{V}_{j m k} \vartheta_{j m}(\rho),
$$

with $\mathcal{U}_{j m k}$ and $\mathcal{V}_{j m k}$ defined by Eqs. (13a) and (13b). Inserting Eq. (B3) and keeping in mind Eq. (B2) [or, in other words, taking $\Delta_{j m, j^{\prime} m}=0$ for $\left.j \neq j^{\prime}\right]$, we can rewrite Eqs. (A3a) and (A3b) in the following form:

$$
\begin{gathered}
{\left[E_{j m k}-T_{j m k}^{(+)}\right] \mathcal{U}_{j m k}=\Delta_{j m} \mathcal{V}_{j m k},} \\
{\left[E_{j m k}+T_{j m k}^{(-)}\right] \mathcal{V}_{j m k}=\Delta_{j m} \mathcal{U}_{j m k} .}
\end{gathered}
$$

A nontrivial solution of these equations exists only when

$$
E_{j m k}= \pm \sqrt{\left(\xi_{j m k}+\frac{\hbar^{2} q^{2}}{2 m_{e}}\right)^{2}+\Delta_{j m}^{2}}+\hbar^{2} k q / m_{e},
$$

with the single-electron energy given by

$$
\xi_{j m k}=\frac{\hbar^{2}}{2 m_{e}}\left[\frac{\alpha_{j m}^{2}}{R^{2}}+k^{2}\right]-\mu .
$$

The sign + in Eq. (B5) corresponds to the physical branch [see discussion in Sec. II, next to Eq. (3)]. Note that the term proportional to $q^{2}$ is canceled due to the renormalization of the chemical potential in the presence of the supercurrent. ${ }^{25}$ Then, the physical solution reads

$$
E_{j m k}=\sqrt{\xi_{j m k}^{2}+\Delta_{j m}^{2}}+\hbar^{2} k q / m_{e} .
$$

It is of importance that for $q>q_{j m}=\frac{m_{e} \Delta_{j m}}{\hbar^{2} k_{j m}}\left[k_{j m}\right.$ is defined by $\left.\left.\xi_{j m k}\right|_{k=k_{j m}}=0\right]$ there exist negative quasiparticle energies. Quasiparticles with negative energies survive in the system even at zero temperature, which means a reconstruction of the ground state due to depairing of electrons [see discussion on Eq. (7) in Ref. 20] in the corresponding single-electron subband. Notice that due to the transverse quantization of the electron motion the set of $k_{j m}$ controls the gapless regime rather than $k_{F}$ (compare with bulk). We also remark that due to the presence of the Doppler term $\hbar^{2} \mathrm{kq} / \mathrm{m}_{e}$ in Eq. (B7), $f_{j m k} \neq f_{j, m,-k}$ in the presence of the longitudinal supercurrent.

For the physical solution, from Eqs. (B4a) and (B4b) one can get [Eq. (14) is also needed]

$$
\begin{gathered}
\mathcal{U}_{j m k} \mathcal{V}_{j m k}=\frac{\Delta_{j m}}{2 \sqrt{\xi_{j m k}^{2}+\Delta_{j m}^{2}}}, \\
\mathcal{V}_{j m k}^{2}=\frac{1}{2}\left(1-\frac{\xi_{j m k}}{\sqrt{\xi_{j m k}^{2}+\Delta_{j m}^{2}}}\right), \\
\mathcal{U}_{j m k}^{2}=\frac{1}{2}\left(1+\frac{\xi_{j m k}}{\sqrt{\xi_{j m k}^{2}+\Delta_{j m}^{2}}}\right) .
\end{gathered}
$$

This allows us to rephrase Eq. (3) as

$$
\Delta_{j^{\prime} m^{\prime}}=\sum_{j m} g_{j^{\prime} m^{\prime}, j m} \mathcal{I}_{j m} \Delta_{j m},
$$

where

$$
\mathcal{I}_{j m}=\frac{1}{8 \pi^{2}} \int_{-\infty}^{+\infty} \mathrm{d} k \frac{\theta\left(\hbar \omega_{D}-\left|\xi_{j m k}\right|\right)}{\sqrt{\xi_{j m k}^{2}+\Delta_{j m}^{2}}}\left(1-2 f_{j m k}\right),
$$

with $\theta()$ the Heaviside step function [see the selection introduced by Eq. (4)] and

$$
g_{j^{\prime} m^{\prime}, j m}=g \int_{0}^{R} \mathrm{~d} \rho \rho \vartheta_{j^{\prime} m^{\prime}}^{2}(\rho) \vartheta_{j m}^{2}(\rho) .
$$

The position-dependent order parameter $\Delta(\rho)$ can be calculated with

$$
\Delta(\rho)=g \sum_{j m} \vartheta_{j m}^{2}(\rho) \mathcal{I}_{j m} \Delta_{j m}
$$

\section{APPENDIX C: SUPERFLUID DENSITY}

We can rewrite Eq. (12) as

$$
j_{s}=|e| n_{e} v_{s}-j_{n},
$$

where Eq. (7) is used and the contribution of the normal component is of the form

$$
j_{n}=\frac{e}{\pi^{2} R^{2}} \sum_{j m} \int_{-\infty}^{+\infty} d k \frac{\hbar k}{m_{e}}\left[\mathcal{U}_{j m k}^{2} f_{j m k}-\mathcal{V}_{j m k}^{2}\left(1-f_{j m k}\right)\right] .
$$

Then, using Eq. (14), we can get 


$$
j_{n}=\frac{e \hbar}{m_{e} \pi^{2} R^{2}} \sum_{j m} \int_{-\infty}^{+\infty} d k k\left[f_{j m k}-\mathcal{V}_{j m k}^{2}\right] .
$$

Equation (C3) can further be simplified due to

$$
\int_{-\infty}^{+\infty} d k k \mathcal{V}_{j m k}^{2}=0
$$

since the coherent factors $\mathcal{U}_{j m k}$ and $\mathcal{V}_{j m k}$ turn out to be independent on the sign of $k$ [see Eq. (B8c)]. Then, we arrive at

$$
j_{n}=\frac{e \hbar}{m_{e} \pi^{2} R^{2}} \sum_{j m} \int_{-\infty}^{+\infty} d k k f_{j m k} .
$$

Notice that $j_{n}>0$ for $v_{s} \neq 0$ because for any given $|k|$ the bogolon occupation number $f_{j m k}$ is larger for negative $k$ [see Eq. (B7)]. Now, when using the representation $j_{s}=|e| n_{s} v_{s}$ [see Eq. (15)], we obtain for the superfluid density

$$
n_{s}=n_{e}+\frac{\hbar}{m_{e} \pi^{2} R^{2} v_{s}} \sum_{j m} \int_{-\infty}^{+\infty} d k k f_{j m k} .
$$

${ }^{1}$ Y. Guo, Y.-F. Zhang, X.-Y. Bao, T.-Z. Han, Z. Tang, L.-X. Zhang, W.-G. Zhu, E. G. Wang, Q. Niu, Z. Q. Qiu, J.-F. Jia, Z.-X. Zhao, and Q. K. Xue, Science 306, 1915 (2004).

${ }^{2}$ M. M. Özer, J. R. Thompson, and H. H. Weitering, Nat. Phys. 2, 173 (2006); M. M. Özer, Y. Jia, Z. Zhang, J. R. Thompson, and H. H. Weitering, Science 316, 1594 (2007).

${ }^{3}$ D. Eom, S. Qin, M.-Y. Chou, and C. K. Shih, Phys. Rev. Lett. 96, 027005 (2006).

${ }^{4}$ M. Savolainen, V. Touboltsev, P. Koppinen, K.-P. Riikonen, and K. Arutyunov, Appl. Phys. A: Mater. Sci. Process. 79, 1769 (2004); M. Zgirski, K.-P. Riikonen, V. Touboltsev, and K. Arutyunov, Nano Lett. 5, 1029 (2005).

${ }^{5}$ M. Tian, J. Wang, J. S. Kurtz, Y. Liu, M. H. W. Chan, T. S. Mayer, and T. E. Mallouk, Phys. Rev. B 71, 104521 (2005).

${ }^{6}$ F. Altomare, A. M. Chang, M. R. Melloch, Y. Hong, and C. W. Tu, Phys. Rev. Lett. 97, 017001 (2006).

${ }^{7}$ L. Janković, D. Gournis, P. N. Trikalitis, I. Arfaoui, T. Cren, P. Rudolf, M.-H. Sage, T. T. M. Palstra, B. Kooi, J. De Hosson, M. A. Karakassides, K. Dimos, A. Moukarika, and T. Bakas, Nano Lett. 6, 1131 (2006).

${ }^{8}$ P. W. Anderson, J. Phys. Chem. Solids 11, 26 (1959).

${ }^{9}$ A. A. Abrikosov and L. P. Gorkov, Zh. Eksp. Teor. Fiz. 36, 319 (1959) [Sov. Phys. JETP 9, 220 (1959)].

${ }^{10}$ B. L. Altshuler, A. G. Aronov, and P. A. Lee, Phys. Rev. Lett. 44, 1288 (1980); B. L. Altshuler, JETP Lett. 41, 648 (1985); P. A. Lee and A. D. Stone, Phys. Rev. Lett. 55, 1622 (1985).

${ }^{11}$ A. I. Larkin, Ann. Phys. 8, 785 (1999); M. A. Skvortsov and M. V. Feigel'man, Phys. Rev. Lett. 95, 057002 (2005).

${ }^{12}$ J. M. Blatt and C. J. Thompson, Phys. Rev. Lett. 10, 332 (1963).

${ }^{13}$ A. A. Shanenko and M. D. Croitoru, Phys. Rev. B 73, 012510 (2006).
${ }^{14}$ M. D. Croitoru, A. A. Shanenko, and F. M. Peeters, Phys. Rev. B 76, 024511 (2007).

${ }^{15}$ Yu. S. Barash and P. I. Nagornyh, JETP Lett. 83, 376 (2006).

${ }^{16}$ V. N. Gladilin, V. M. Fomin, and J. T. Devreese, Solid State Commun. 121, 519 (2002).

${ }^{17}$ A. A. Shanenko, M. D. Croitoru, M. Zgirski, F. M. Peeters, and K. Arutyunov, Phys. Rev. B 74, 052502 (2006).

${ }^{18}$ A. A. Shanenko, M. D. Croitoru, and F. M. Peeters, Europhys. Lett. 76, 498 (2006); Phys. Rev. B 75, 014519 (2007).

${ }^{19}$ J. E. Han and V. H. Crespi, Phys. Rev. B 69, 214526 (2004).

${ }^{20}$ A. A. Shanenko, M. D. Croitoru, and F. M. Peeters, Phys. Rev. B 78, 024505 (2008).

${ }^{21}$ L. Landau, J. Phys. (USSR) 5, 71 (1941).

${ }^{22}$ J. Bardeen, Rev. Mod. Phys. 34, 667 (1962).

${ }^{23}$ P. F. Bagwell, Phys. Rev. B 49, 6841 (1994).

${ }^{24}$ P. G. de Gennes, Superconductivity of Metals and Alloys (Benjamin, New York, 1966).

${ }^{25}$ A. V. Swidzinsky, Spatially Inhomogeneous Problems in the Theory of Superconductivity (Nauka, Moscow, 1982).

${ }^{26}$ A. M. Zagoskin, Quantum Theory of Many-Body Systems: Techniques and Applications (Springer-Verlag, New York, 1998).

${ }^{27}$ A. L. Fetter and J. D. Walecka, Quantum Theory of ManyParticle Systems (Dover, New York, 2003).

${ }^{28}$ D. G. Naugle, J. W. Baker, and R. E. Allen, Phys. Rev. B 7, 3028 (1973).

${ }^{29}$ F. M. Peeters, M. D. Croitoru, and A. A. Shanenko, Physica C 468, 326 (2008); A. A. Shanenko, M. D. Croitoru, and F. M. Peeters, ibid. 468, 593 (2008).

${ }^{30}$ A. Kohen, T. Proslier, T. Cren, Y. Noat, W. Sacks, H. Berger, and D. Roditchev, Phys. Rev. Lett. 97, 027001 (2006). 